\section{¿Qué significa la federalización de la ciencia y la tecnología en Argentina?}

Resumen: En la última década, la federalización de la ciencia y la tecnología (CyT) se ha establecido como idea fuerza en Argentina, tanto en la agenda como en el discurso de políticas para el área. Sin embargo, el concepto mismo de federalización resulta un tanto difuso y en ocasiones se ha utilizado de forma ambigua. A partir de una revisión de estudios académicos previos, documentos institucionales y expresiones de diferentes actores del sistema, este trabajo se propone ordenar las ideas que se manejan respecto de este tema, principalmente aquéllas surgidas de debates ocurridos durante la última década. En este contexto se distinguen dos concepciones diferentes: desconcentración y descentralización. Esta distinción puede resultar de utilidad a la hora de definir y clarificar líneas de acción para establecer políticas CyT integradas de alcance nacional, regional y provincial.

Palabras claves: Políticas de ciencia y tecnología, desigualdades territoriales, desconcentración, descentralización.

What does the federalization of science and technology mean in Argentina?

Abstract: In the last decade, the federalization of science and technology (S\&T) has established itself as a key force in Argentina, both in the agenda and the policy discourses for the area. However, the concept of federalization is, to a certain extent, vague and has sometimes been used ambiguously. Based on a review of previous academic studies, institutional documents and expressions of different actors, this article aims at organizing the most common ideas on this topic, especially those that arose out of debates during the last decade. In this context, two different approaches are distinguished: deconcentration and decentralization. This distinction can be useful when defining and clarifying lines of action to establish integrated S\&T policies at a national, regional and provincial level.

Keywords: Science and Technology Policies, Territorial Inequalities, Deconcentration, Decentralization.

\section{O que significa a federalização da ciência e tecnologia na Argentina?}

Resumo: Na última década, a federalização da ciência e tecnologia (C\&T) estabeleceuse como uma forte idéia na Argentina, tanto na agenda quanto no discursos políticos da área. No entanto, o próprio conceito de federalização é um tanto vago e às vezes tem sido usado de forma ambígua. Com base em uma revisão de estudos acadêmicos anteriores, documentos institucionais e expressões de diferentes atores do sistema, este trabalho tem como objetivo ordenar as idéias que são tratadas com relação a esse tópico, principalmente aquelas que surgem de debates que ocorreram durante a última década. Nesse contexto, distinguem-se duas concepções diferentes: desconcentração e descentralização. Essa distinção pode ser útil ao definir e esclarecer linhas de ação para estabelecer políticas integradas de C\&T de âmbito nacional, regional e provincial.

Palavras-chave: Políticas de ciência e tecnologia, desigualdades territoriais, desconcentração, descentralização.

\section{Ciencia Tecnología y Política \\ Año 3 N 4 Mayo 2020}

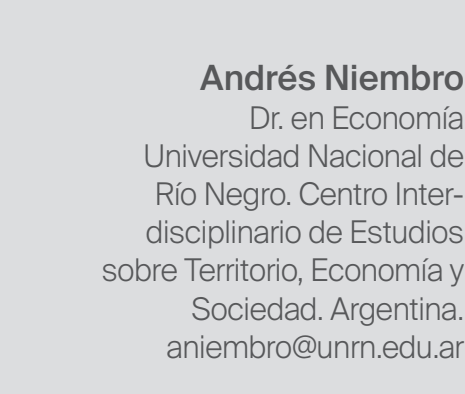

Año 3 N $^{\circ} 4$ Mayo 2020

Fecha de recibido: $27 / 02 / 2020$

Fecha de aprobado: 23/04/2020

https://doi.org/10.24215/26183188e036

https://revistas.un|p.edu.ar/CTyP

ISSN 2618-3188

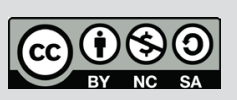

Esta obra está bajo licencia Creative Commons Atribución-NoComercial-Compartirlgual 4.0 Internacional http://creativecommons.org/licenses/bync-sa/4.O/deed.es_AR 
| Ciencia, Tecnología y Política | Año 3 | N4 | Mayo 2020 | ISSN 2618-3188 | www.revistas.unlp.edu.ar/CTyP |

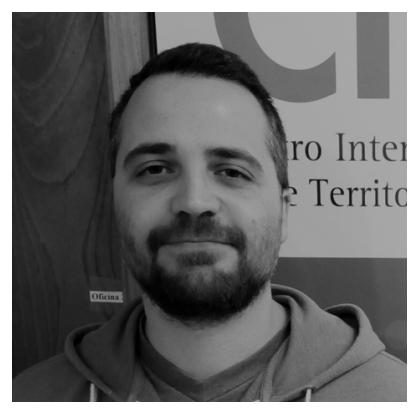

Andrés Niembro

Dr. en Economía

Universidad Nacional de Río Negro. Centro Interdisciplinario de Estudios sobre Territorio, Economía y Sociedad. Argentina. aniembro@unrn.edu.ar

\section{¿Qué significa la federalización de la ciencia y la tecnología en Argentina?}

Resumen: En la última década, la federalización de la ciencia y la tecnología (CyT) se ha establecido como idea fuerza en Argentina, tanto en la agenda como en el discurso de políticas para el área. Sin embargo, el concepto mismo de federalización resulta un tanto difuso y en ocasiones se ha utilizado de forma ambigua. A partir de una revisión de estudios académicos previos, documentos institucionales y expresiones de diferentes actores del sistema, este trabajo se propone ordenar las ideas que se manejan respecto de este tema, principalmente aquéllas surgidas de debates ocurridos durante la última década. En este contexto se distinguen dos concepciones diferentes: desconcentración

descentralización. Esta distinción puede resultar de utilidad a la hora de definir y clarificar líneas de acción para establecer políticas CyT integradas de alcance nacional, regional y provincial.

\section{Introducción}

Si bien el objetivo de la federalización de la ciencia y la tecnología (CyT) ha estado presente en varios momentos de la historia argentina, en la última década se ha establecido como idea fuerza, tanto en la agenda como en el discurso de políticas para el área. Esto ha ocurrido en el caso del Ministerio de Ciencia, Tecnología e Innovación Productiva (MINCyT) a partir de 2007, al igual que en algunos de los principales organismos nacionales de CyT, como por ejemplo en el Consejo Nacional de Investigaciones Científicas y Técnicas (CONICET) (Szpeiner y Jeppesen, 2013). Otro aspecto a destacar es que la preocupación por la federalización pareciera haber trascendido, al menos técnicamente, a los últimos cambios de gobierno a nivel nacional (a fines de 2015 y de 2019), más allá de las profundas diferencias políticas y de gestión que hubo en las respectivas administraciones. En efecto, a pesar de las modificaciones organizacionales ocurridas en los 
últimos años -primero con la eliminación del MINCyT y su conversión en Secretaría a fines de 2018 y recientemente con su recuperación de nuevo como Ministerio-, ha subsistido dentro del organigrama del área la sub/secretaría de federalización de la CyT. Asimismo, el último informe público de gestión del CONICET destaca que para fortalecer las capacidades en CyT "se pone el énfasis en la promoción de la federalización” (CONICET, 2018: 11).

Por otro lado, durante 2019 la noción de la federalización de la CyT parece haberse popularizado en el albor de diferentes encuentros (federales), discusiones y debates políticos previos a las elecciones nacionales y, luego de las primarias de agosto, descontando un inminente cambio de gobierno y gestión para el área. Lo interesante, nuevamente, es que pareciera haber cierto consenso político en la necesidad de avanzar y profundizar la federalización de la CyT en Argentina.

Detrás de este principio de acuerdo, es de suponer que también podría encontrarse un diagnóstico medianamente compartido: que la Argentina es un país con profundas desigualdades territoriales; que las mismas a su vez se han manifestado históricamente en el sistema de CyT; y que en la última década los progresos en materia de federalización han sido modestos, incipientes y dispares entre regiones o provincias, como lo demuestran diferentes estudios sobre el tema (Gallardo, 2015; Jeppesen et al., 2015; Niembro, 2020; Sarthou, 2019; Suárez y Fiorentin, 2018). Esto mismo es señalado por diferentes actores claves del complejo de CyT argentino. Por ejemplo, Dora Barrancos, quien formó parte del Directorio del CONICET entre 2010 y 2019, reconocía que "una cuenta pendiente es profundizar la federalización del sistema"1. Por otro lado, el actual Ministro de Ciencia,
Tecnología e Innovación (CTI), Roberto Salvarezza, señalaba recientemente que "las asimetrías estructurales que existen entre las regiones de nuestro país precisan un desarrollo científico y tecnológico realmente federal".

En este marco de popularización de la idea de federalización de la CyT, es preciso reconocer que el concepto mismo de federalización resulta un tanto difuso y que en ocasiones el término se ha utilizado de forma liviana o imprecisa. Por ello, creemos que es posible (y necesario) realizar algunos aportes preliminares para empezar a aclarar un poco más el panorama sobre una pregunta central: ¿qué significa (o qué se entiende por) la federalización de la CyT en Argentina?

En este trabajo se intentará, por un lado, ordenar las ideas que se manejan respecto de este tema, principalmente aquéllas surgidas de debates ocurridos durante la última década. Por otro lado, se busca definir un conjunto de bases conceptuales que permitan, a futuro, construir líneas de acción para establecer políticas integradas de alcance nacional, regional y provincial. En términos prácticos, si bien existen algunos estudios académicos previos que abordaron diferentes aspectos de la federalización, los antecedentes más recientes se encuentran reflejados sobre todo en diversos documentos institucionales o expresiones de actores claves en distintos medios de comunicación. Por lo tanto, el trabajo se sustentará, especialmente, en la búsqueda, procesamiento y análisis de estas fuentes.

En las siguientes secciones se diferenciarán dos enfoques o miradas respecto a la federalización: 1) Un enfoque que podríamos llamar cuantitativo, ligado principalmente a la desconcentración territorial de la inversión y el personal en CyT y, con ello, a poder

\footnotetext{
1 Por una cuestión de extensión y para mayor simplicidad del artículo, las referencias a las notas periodísticas de donde se derivan las afirmaciones de actores claves, pueden consultarse en un Documento de Trabajo más extenso, disponible en: http://cietes.unrn.edu.ar/images/Niembro_WP2020_01_vf.pdf. Vale destacar que, en todos los casos, los resaltados en cursiva corresponden al autor.
} 
lograr una distribución regional-provincial más equitativa; y 2) Un enfoque más de tipo cualitativo o, si se quiere, institucional -aunque el primer enfoque también implica cambios institucionales obviamente-, vinculado a la descentralización de algunas decisiones y/o funciones en torno a los instrumentos y políticas de CTI. El artículo se completa con algunas reflexiones finales y posibles temáticas sobre las que se debería profundizar a futuro.

\section{La federalización como desconcentración}

El enfoque de la federalización de la CyT pensada como desconcentración pareciera ser la mirada más tradicional sobre esta problemática en Argentina. El mismo está fundado y ha sido una respuesta a la histórica concentración territorial del sistema de CyT en unas pocas ciudades y provincias del país -generalmente las más desarrolladas de la zona central o pampeana-. Esta mirada puede rastrearse, incluso, en los últimos planes nacionales de CTI, donde se destaca la marcada concentración de recursos en la Ciudad y Provincia de Buenos Aires, en Córdoba y en Santa Fe, y los fuertes desbalances territoriales que esta realidad conlleva (MINCyT, 2011 y 2013; SECyT, 2005). Asimismo, podemos encontrar allí diferentes objetivos numéricos a nivel territorial. En primer lugar, tanto en las "Bases para un Plan Estratégico de Mediano Plazo en Ciencia, Tecnología e Innovación" (SECyT, 2005) como en el "Plan Nacional de Ciencia, Tecnología e Innovación 20122015" (MINCyT, 2011) se establecía la meta para el año 2015 de "duplicar la participación, en el total de recursos de $I+D$, de las diecinueve provincias que explicaban en conjunto algo menos del $20 \%$ del total" (MINCyT, 2011: 41) -es decir, llevarlo al 40\%-.
No obstante, en el posterior "Plan Argentina Innovadora 2020" (MINCyT, 2013) este objetivo se redujo a alcanzar un 32\% en 2015 y 37\% en 2020. A su vez, en este último plan se señalaba que: "La necesidad de articular territorialmente se enmarca en el reconocimiento de la concentración regional de las actividades de CTI. Avanzar hacia una distribución más equitativa a nivel territorial contribuiría, en consecuencia, a apoyar procesos de convergencia socioeconómica regional" (MINCyT, 2013: 47).

Por otro lado, existe amplia evidencia de este patrón de concentración territorial a lo largo de diferentes componentes del sistema de CyT, como por ejemplo en el caso del CONICET (Gallardo, 2015; Jeppesen et al., 2015; Niembro, 2020; Sarthou, 2019; Szpeiner y Jeppesen, 2013), del sistema universitario (Lugones et al., 2010; Moldovan et al., 2011)², o del destino geográfico de los distintos fondos de promoción que otorgó hasta 2019 la Agencia Nacional de Promoción Científica y Tecnológica -ANPCYT- (Peirano, 2011; Sarthou, 2019; Suárez y Fiorentin, 2018).

Algo similar se puede apreciar por el lado del Instituto Nacional de Tecnología Industrial (INTI). Frente al diagnóstico de una marcada concentración en el área central del país, desde mediados de los 2000 se ha apuntado a mejorar la presencia del INTI con al menos un centro de investigación y desarrollo en cada una de las provincias argentinas (Solanas, 2009). Según otros documentos institucionales, durante la última década se continuó "con la estrategia de federalización del INTI para obtener una mayor cobertura territorial de los servicios a prestar, a lo largo y ancho del territorio" (ONP, 2016: 3). En cambio, la historia del Instituto Nacional de Tecnología Agropecuaria (INTA) muestra que la "arquitectura admi-

\footnotetext{
2 Si bien en los últimos años se han creado varias universidades públicas nuevas en el interior del país, en paralelo se ha expandido considerablemente la cantidad de instituciones en el conurbano bonaerense (González y Claverie, 2017; Marquina y Chiroleu, 2015). Como señalan Beigel et al. (2018), muchas de estas universidades están estrechamente conectadas con la ciudad de Buenos Aires, tanto por la migración de docentes y/o investigadores formados en la Universidad de Buenos Aires (UBA) como por tener diferentes tipos de instalaciones (de posgrado y/o investigación) en la CABA.
} 
nistrativa fue dando forma a un modo de operar sobre la totalidad del territorio nacional con un carácter decididamente federal", por lo que "la federalización de INTA es literal, alcanza rincones del país, como pocas dependencias gubernamentales lo hacen" (Iparraguirre, 2017: 192-193).

El caso del CONICET es clave, pues, en cierta medida, ha operado como la punta de lanza de la expansión del sistema argentino de CyT en los últimos años (Niembro, 2020). En 2017, el por entonces Presidente del CONICET, Alejandro Cecatto, manifestaba que:

Federalizar el sistema científico argentino es una gran necesidad, por una cuestión de equidad. El $75-80 \%$ de los recursos humanos del CONICET están concentrados en la zona central del país $y$ hay grandes esfuerzos que hacen grupos incipientes (...) en distintas regiones más alejadas, desde la Patagonia, Noroeste o Noreste del país. Entonces, hemos tomado como decisión del Directorio del CONICET, una decisión importante, muy proactiva en cuanto a la federalización, que ha sido llevar a un 30\% el ingreso a carrera del investigador con posiciones asignadas a estas zonas de vacancia geográfica (...) y pretendemos seguir avanzando en la medida que esos cupos vayan generando una distribución más equitativa.

Este último porcentaje es un poco superior al que se planteaba originalmente en el llamado "Plan Barañao 2013" (en alusión al por entonces Ministro de CTI), donde se señalaba que "se apuntará a la federalización de los recursos humanos priorizando el $25 \%$ de las vacantes del CONICET en aquellas regiones del país con escaso desarrollo científico"
(Hurtado, 2016). El propio Barañao hacía referencia en 2017 a la "federalización de la ciencia, mediante la cual tratamos de corregir una asimetría muy grande respecto a la distribución de doctores, en su gran mayoría localizados en las grandes ciudades, dado que el 60\% está entre la Ciudad y la Provincia de Buenos Aires". En función de todas estas manifestaciones, puede entenderse por qué consideramos a este abordaje de la federalización como una mirada mayormente de naturaleza cuantitativa.

En tanto, la actual Presidenta del CONICET, Ana Franchi, fijaba recientemente entre sus prioridades "federalizar ese organismo para que no exista concentración sólo en las grandes ciudades y bajar la ciencia al territorio, una acción que permite resolver los problemas del país". Menciones similares sobre la concentración territorial del sistema de СyT pueden encontrarse en numerosas notas, documentos e incluso en algunas recomendaciones de política CyT (Abeledo et al., 2015; CLCPyS, 2019; Laborde y Del Bono, 2019).

En definitiva, la federalización de la CyT en tanto desconcentración apunta a una redistribución regional de los recursos con vistas a una mayor equidad territorial. Otra manifestación común es la búsqueda de un desarrollo equilibrado y armónico del sistema de CyT en todo el país (CLCPyS, 2019; Szpeiner y Jeppesen, 2013). Entre los borradores de la "Estrategia Nacional de CTI Argentina Innovadora 2030" (SECyT, 2019)³ se plantea la necesidad de lograr "menores desequilibrios regionales en cuanto a capacidades y recursos" y una "disminución de la brecha estructural entre las regiones".

\footnotetext{
3 Son los borradores de un nuevo plan de CTI que vendría a suceder al "Argentina Innovadora 2020" y que fue discutido durante la Presidencia de Macri. No obstante, esta nueva "Estrategia 2030" ha recibido varias críticas y nunca llegó a publicarse oficialmente. Como se trata de un conjunto de archivos preliminares (separados) y no de un documento general e integrador, se volvería engorroso referenciar las páginas concretas de algunas citas.
} 
La federalización como descentralización

Si bien el enfoque de la federalización como desconcentración ha sido muy común en Argentina, en paralelo pueden encontrarse expresiones recientes ligadas a la descentralización ${ }^{4}$, con una mirada más de corte institucional. No obstante, es preciso realizar algunas aclaraciones.

En primer lugar, puesto que los fondos y el personal de CyT tienden a concentrarse en torno a la zona central del país (la CABA y las provincias de Buenos Aires, Córdoba y Santa Fe), en algunos casos descentralizar podría interpretarse erróneamente como redistribuir estos recursos desde la zona centro hacia otras regiones. En 2016, quien fuera Secretario de Articulación del MINCyT, Agustín Campero, sostenía que "le queremos dar un impulso muy fuerte a la federalización, ya que la ciencia y la tecnología en la Argentina están muy centralizadas, siguen la tendencia de la distribución de los habitantes y de la riqueza económica". El propio Salvarezza, en una entrevista de 2017 y frente a la reducción del cupo de ingresos a carrera del CONICET, señalaba que "en un sistema donde prima la meritocracia, va a ganar el más fuerte" y "esta competencia darwiniana va a acrecentar la centralización". En una nota reciente, titulada "Tucumán llamó a federalizar los fondos nacionales para la actividad científica", se plantea que el Secretario del área en dicha provincia, Mariano Garmendia, "pidió descentralizar los recursos concentrados en cuatro distritos". En función de los términos planteados en este trabajo, en estas declaraciones lo correcto hubiera sido hablar de desconcentración, desde la zona central hacia las demás.

En segundo lugar, la descentralización puede contener tranquilamente un componente de desconcen- tración territorial en términos organizacionales. Un ejemplo de ello es la descentralización de algunas funciones administrativas y de gestión del CONICET a partir de la creación de Centros Científicos Tecnológicos (CCT) en distintas regiones (González, 2017).

Por último, teniendo en cuenta esta perspectiva organizacional-institucional, es preciso recuperar por un momento el origen de términos como federal, federalismo o federalizar. Sin caer en grandes tecnicismos y debates, podemos definir al federalismo como un sistema político en el cual los poderes y funciones de gobierno se distribuyen entre un conjunto de Estados asociados (las provincias), que conservan cierto grado de autonomía en el ejercicio de algunas competencias, y un Estado federal, central o nacional, en el cual se delegan otras atribuciones y poderes. El punto clave es cómo se establece dicha división de poderes y dónde se ponen los límites de competencias entre el nivel provincial y el central-nacional. En este sentido, como plantean Rodríguez Reyes y Meza Medina (2011), el término federalizar puede presentar diferentes acepciones. Si se trata de un sistema ya descentralizado o no centralizado -es decir, con funciones y poderes del lado de las provincias-, la federalización podría tomarse como sinónimo de centralización, transfiriendo al menos parte de esas competencias hacia el Estado federal-central-nacional. En cambio, si el sistema se encuentra centralizado, federalizarlo implicaría un proceso de descentralización.

De igual forma, Dalla Vía (2002: 58) señala que "el sistema federal no siempre supone un proceso de descentralización territorial". Por el contrario, en los Estados Unidos se desarrolló un proceso de centralización de funciones y competencias desde los Estados locales preexistentes hacia el Estado fe-

\footnotetext{
4 Un ejemplo elocuente fue el panel sobre "La Descentralización y Federalización de la Ciencia y la Tecnología en Argentina", en el marco de las "Jornadas Federales de Ciencias y Tecnologías para el Desarrollo Nacional", realizadas los primeros días de octubre de 2019 en Resistencia, Chaco.
} 
deral. En Argentina, el federalismo se adoptó en un contexto diferente, como una forma de superar los enfrentamientos entre unitarios y federales, aunque estos últimos no eran estrictamente federalistas en el sentido norteamericano (Dalla Vía, 2002; Granato, 2015). Como describe este último autor, "la Argentina es una federación descentralizada en la que los gobiernos provinciales son políticamente autónomos, pero financieramente dependientes, que conviven con un presidencialismo fuerte, con una alta concentración geográfica de recursos productivos y con amplias desigualdades interprovinciales" (Granato, 2015: 119). Por ello también, "la interpretación común [es] que federalizar es descentralizar" (Ruarte Bazán, 2009: 2).

El diseño y financiamiento de las políticas de CTI en Argentina han mostrado históricamente un carácter centralizado, por lo que las políticas, los instrumentos y los fondos de CTI suelen llegar al territorio a partir de una lógica "de arriba abajo" (top-down), desde el plano central-nacional (Barceló et al., 2015; González, 2017; Yoguel et al., 2005). En tanto, el presupuesto asignado a nivel provincial para la función de ciencia y técnica suele ser marginal (Niosi, 2013; Zurbriggen y González Lago, 2010), ya que los esfuerzos de las provincias tienden a concentrarse en otras funciones principales -varias de ellas, descentralizadas hace algunas décadas-, como salud pública, educación básica, seguridad y justicia, entre otras.

Si bien ésta es una realidad en buena medida compartida con otros países de América Latina, en los últimos años pueden encontrarse también algunos intentos de descentralización de las políticas de CTI, aunque con intensidades y velocidades diferentes (Llisterri y Pietrobelli, 2011; Rivas et al., 2014). El caso de Brasil, por ejemplo, se ha tomado como una experiencia interesante para extraer lecciones al res- pecto (Albornoz et al., 2015; CEPAL, 2017). En tanto, en Europa las discusiones sobre políticas regionales de innovación han crecido sostenidamente en las últimas décadas (González-López et al., 2019; Isaksen et al., 2018) e incluso se han desplegado algunas consultorías para transferir o promover políticas de innovación descentralizadas en Latinoamérica (Barceló et al., 2015; INFYDE, 2018).

En Argentina, como destaca González (2017: 218), "las relaciones institucionales de la estructura de ciencia y tecnología pueden caracterizarse por la preeminencia de instancias centralizadas". La autora resalta también que, siguiendo con un patrón topdown, "el factor de mayor dinamismo para impulsar iniciativas de territorialización está asociado al impulso del gobierno central" (González, 2017: 217). En otros términos, las iniciativas de federalización de la CyT en Argentina suelen arrancar (y sostenerse o discontinuarse) mayormente por interés del gobierno nacional.

La prioridad que le conceden los gobiernos provinciales a la CyT dentro de la agenda pública es bastante heterogénea. Además de la baja incidencia presupuestaria en relación con otras áreas, como educación y salud, el rango asignado a esta cartera en las provincias suele ser también bajo. Luego de los cambios de mandato de diciembre de 2019, son muy pocas las provincias con Ministerios de CTI y en algunas, como Buenos Aires y Santa Fe, incluso se ha reducido el rango. En la mayoría de los casos se trata de Secretarías o Subsecretarías, que dependen del Ministerio de Educación o en algunas provincias, como Buenos Aires, del ala de Industria-Producción (Ministerio de Producción, Ciencia e Innovación Tecnológica de la Provincia de Buenos Aires) ${ }^{5}$.

En general, son pocas (aunque no inexistentes) las manifestaciones que, entendiendo a la federaliza-

\footnotetext{
5 Ver en este mismo número el artículo de la Cátedra Libre CPS sobre Ciencia y Tecnología en la Provincia de Buenos Aires.
} 
ción de la CyT como descentralización, llegan a abogar por una autonomía provincial en la materia. Por ejemplo, el Secretario del área en Tucumán, Mariano Garmendia, recientemente "consideró necesario que las provincias consoliden procesos de autonomización de la gestión nacional, en el sector de ciencia y tecnología", lo cual se refleja en la idea de constituir "en las provincias Agencias de Ciencia y Tecnología para recibir recursos de una manera más ágil y que las provincias tengan capacidad autónoma".

Un paso más allá parecen ir las recomendaciones de Niosi (2013). En su ejercicio comparativo con el caso de Canadá, el autor reconoce que en Argentina no sólo el sistema de CyT se encuentra altamente centralizado, sino también el sistema fiscal -es decir, la recaudación de impuestos-. De allí, sugiere que "Argentina necesita descentralizar los ingresos del gobierno para permitir que las provincias desarrollen sus propias políticas en ICT [léase CTI]" (Niosi, 2013: 344). Sin entrar en un debate que excede a los alcances de este artículo y que hemos dado brevemente en otro trabajo (Niembro, 2019), podríamos simplemente recurrir a una asociación lógica y decir que, puesto que lo primero es altamente improbable que ocurra en términos políticos (o incluso contraproducente) ${ }^{6}$, entonces lo segundo tiende a ser inviable en los hechos.

Dicho esto, podría resultar mucho más factible mejorar las estrategias de descentralización de las políticas nacionales de CTI, sobre la base de las experiencias ya existentes y los aprendizajes obtenidos (Niembro, 2019). En este sentido parece apuntar la "Declaración de las provincias sobre Ciencia, Tecnología e Innovación" de julio de 2019, en la búsqueda por un "Acuerdo Federal para el Diseño y Gestión de las Políticas Públicas de Ciencia, Tecnología e Innovación". Frente a "la imperiosa necesidad de una política federal de ciencia, tecnología e innovación", se propone "ampliar y fortalecer la participación de las provincias en el diseño de la política científica nacional". De forma similar, Josefina Vaca (investigadora del CIAP-UBA y miembro de la comisión de CyT del Instituto Patria) plantea que es preciso "profundizar la federalización de la ciencia, la tecnología y la innovación, a través de la participación de las instancias de gobierno subnacionales en la definición y orientación de políticas". Según Javier Tineo, Secretario de CTI de La Rioja, esto "es relevante porque hay una agenda en la provincia en materia de innovación que no es idéntica a la que maneja el CONICET que es nacional". No obstante, dado que el contexto de las áreas de CTI provinciales es heterogéneo y con márgenes de maniobra en general reducidos, según Josefina Vaca, otro aspecto que "resulta crucial [es] el fortalecimiento de las áreas de CyT sub-nacionales", una cuestión que también es señalada entre los borradores de la "Estrategia 2030" (SECyT, 2019).

En línea con lo anterior, en los últimos tiempos también se ha planteado la necesidad de reformular o fortalecer el rol del Consejo Federal de Ciencia y Tecnología (COFECYT), un organismo que data de 1997 pero que cuenta con antecedentes directos desde inicios de los años noventa (con el COFEA y luego el COFEACYT). En los borradores de la "Estrategia 2030" se menciona que, si bien a través del COFECYT se han logrado avances para una mayor articulación del sistema, "el desafío es profundizar estas iniciativas", entre otros aspectos, fortaleciendo al Consejo "como ámbito de formulación de políticas para constituirse en un actor protagónico de la política científica y tecnológica nacional" (SECyT,

\footnotetext{
${ }^{6}$ La descentralización de ingresos -es decir, de la recaudación de impuestos- tranquilamente podría conducir con el tiempo hacia un ensanchamiento más que a una reducción de las asimetrías provinciales: las jurisdicciones menos desarrolladas (más pobres) recaudarían menos y, por ende, podrían invertir menos en políticas de CTI (o en sus otras funciones), perpetuando así la trampa de pobreza y el círculo vicioso; y viceversa, para el caso de las provincias más desarrolladas (ricas).
} 
2019). Asimismo, entre sus ejes de gestión, el Ministro Salvarezza señalaba recientemente: "Para avanzar en la federalización vamos a fortalecer el lugar del Consejo Federal de Ciencia y Tecnología con el objetivo de promover el desarrollo armónico de las capacidades científicas y tecnológicas en todas las regiones del país". Diego Hurtado, actual Secretario de Planeamiento y Políticas de CTI, también ha destacado que "El COFECYT es el recurso formal que nos va a ayudar a impulsar una política coordinada de federalización".

\section{Reflexiones finales}

A lo largo de este trabajo hemos intentado ordenar la discusión reciente sobre la federalización de la CyT en Argentina, poniendo en evidencia dos concepciones distintas al respecto: desconcentración y descentralización. En parte, el término federalización se ha utilizado a veces de forma ambigua porque no han estado del todo claros o no se han explicitado los objetivos a alcanzar. En este contexto, varios estudios han intentado evaluar, con diferentes criterios, los alcances reales de la federalización en distintos ámbitos del sistema de CyT (Jeppesen et al., 2015; Niembro, 2020; Sarthou, 2019; Suárez y Fiorentin, 2018; Szpeiner y Jeppesen, 2013).

De cara al seguimiento y mejora de las iniciativas de federalización de la CyT en Argentina, la evaluación de dichas políticas es una cuestión clave a profundizar y para ello se necesita una definición clara y explícita de los objetivos en torno a la federalización: ¿se está hablando de desconcentración, de descentralización o de una combinación de ambas? y ¿con qué intensidad o graduación? Muchos de los objetivos cuantitativos planteados en los últimos años resultaron ambiciosos (incluso con el recorte del porcentaje en los planes de CTI) y en cierta medida inalcanzables en el corto plazo. Parte de ello se debe a la falta de correlato entre estos objetivos y las iniciativas y acciones desplegadas realmente, las cuales no modificaron profundamente las reglas del juego del sistema de CyT y, con ello, tampoco su lógica de reproducción circular y concentración territorial en unas pocas ciudades y provincias.

Dado que el concepto de federalización tiene per se una naturaleza dinámica y no estática, cabe preguntarse cómo se planteará la relación entre desconcentración y descentralización. En caso de que la estrategia de federalización incluya a ambas facetas, ¿se avanzará primero con una y luego con otra? y ¿con qué plazos?, o ¿se trabajará sobre las dos dimensiones a la par?

Estas cuestiones requieren, sin duda, una mayor profundización en la temática, al igual que un repaso más completo de la historia (no sólo reciente) acerca de la federalización de la CyT en Argentina. Asimismo, es necesario un mayor contraste entre los discursos y los planes del área frente a los hechos consumados en la realidad y, a partir de estos análisis, una discusión focalizada de políticas (integrales) de CTI que puedan contribuir a la federalización. Todas estas cuestiones serán materia de próximos trabajos.

\section{Agradecimientos}

Se agradece a Rolando González-José la facilitación de algunos documentos, como así también los valiosos comentarios y sugerencias de Nerina Sarthou, Francisco Aristimuño, Juan Carlos Del Bello y los editores de la revista. Como es usual, los errores remanentes son de mi exclusiva responsabilidad.

\section{Bibliografía}

Abeledo, C., Charreau, E., De la Cruz, F., Del Bello, J. C., Lattuada, M. y Mariscotti, M. (2015). Bases para una política de estado en Ciencia, Tecnología e Innovación (CTI). Debate Universitario, 4(7), pp. 87-92. 
Albornoz, M., Barrere, R., Sánchez Macchioli, P., Osorio, L. y Turkenich, M. (2015). Políticas CTI en países emergentes. Análisis comparado de experiencias heterogéneas y su aplicabilidad en Argentina. Informe de Proyecto de Investigación CIECTI-MINCyT.

Barceló, M., Abel, l., Fuster, E., Marrugat, N., Ordenes, A., Hernández, N., . . Sánchez, D. (2015). EU-Latin America cooperation on regional innovation strategies in the framework of regional policy. EU-LAC Foundation, European Union.

Beigel, F., Gallardo, O. y Bekerman, F. (2018). Institutional expansion and scientific development in the periphery: The structural heterogeneity of Argentina's academic field. Minerva, 56(3), pp. 305-331.

CEPAL (2017). Instituciones, políticas e instrumentos para impulsar la ciencia, tecnología e innovación en la Argentina: Reflexiones a partir de la experiencia brasileña. Santiago de Chile: CEPAL, Naciones Unidas.

CLCPyS (Cátedra Libre Ciencia, Política y Sociedad) (2019). ¿Qué hacer con la ciencia y la tecnología en la recuperación del país? Veinte propuestas para el futuro gobierno. Ciencia, Tecnología y Política, 2(2), pp. 1-8.

CONICET (2018). Informe de Gestión al 31-12-2018. Buenos Aires: CONICET.

Dalla Vía, A. (2002). Actualidad del federalismo argentino. En J. M. Serna (Ed.), Federalismo y regionalismo. México DF: UNAM.

Gallardo, O. (2015). Trayectorias de formación de investigadores del CONICET. Revista Sociedad, 34, pp. 121-139.

González-López, M., Asheim, B. y Sánchez-Carreira, M. C. (2019). New insights on regional innovation policies. Innovation: The European Journal of Social Science, 32(1), pp. 1-7.

González, G. (2017). Federalización de la ciencia y la tecnología en Argentina. Una revisión de iniciativas de territorialización y planificación regional (19962007). Revista de Estudios Regionales, 108, pp. 193225.

González, G. y Claverie, J. A. (2017). Planeamiento de la educación superior en Argentina: Entre las políticas de regionalización y los procesos de innovación universitaria (1995-2015). Education Policy Analysis Archives, 25(70), pp. 1-33.

Granato, L. (2015). Federalismo argentino y descentralización: Sus implicancias para la formulación de políticas públicas. Prolegómenos. Derechos y Valores, 18(36), pp. 117-134.

Hurtado, D. (2016). Ciencia para qué. Revista Anfibia. Disponible en: http://revistaanfibia.com/ensayo/ ciencia-para-que/.

INFYDE (2018). EU-CELAC INNOV-AL Platform. Promotion of Decentralised Innovation Policies in CELAC Countries. Work Plan. Disponible en: https:// ec.europa.eu/regional_policy/sources/cooperate/international/innov-al/innov_al_celac_workplan_es.pdf. Iparraguirre, G. (2017). Imaginarios del desarrollo: Gestión política y científica de la cultura. Buenos Aires: Biblos.

Isaksen, A., Martin, R. y Trippl, M. (2018). New avenues for regional innovation systems and policy. En A. Isaksen, R. Martin y M. Trippl (Eds.), New avenues for regional innovation systems - Theoretical advances, empirical cases and policy lessons. Nueva York: Springer.

Jeppesen, C., Goldberg, M., Szpeiner, A. y Rodríguez Gauna, M. C. (2015). Estrategias, instrumentos y resultados de la política pública de RRHH en los últimos diez años. Revista Sociedad, 34, pp. 68-84.

Laborde, M. y Del Bono, T. (2019). Propuestas para comenzar a solucionar algunos de los principales problemas que afectan al CONICET. Buenos Aires: 
| Ciencia, Tecnología y Política | Año 3 | N4 | Mayo 2020 | ISSN 2618-3188 | www.revistas.unlp.edu.ar/CTyP |

CONICET. Mimeo.

Lugones, G., Hurtado, D., Gutti, P., Mallo, E., Bázque, H. y Alonso, M. (2010). El rol de las universidades en el desarrollo científico y tecnológico en la década 1998-2007: Informe Nacional Argentina. Documento de Proyecto. Santiago de Chile: Centro Interuniversitario de Desarrollo (CINDA) - Universia.

Llisterri, J. J. y Pietrobelli, C. (2011). Los sistemas regionales de innovación: Conclusiones y recomendaciones. En J. J. Llisterri y C. Pietrobelli (Eds.), Los sistemas regionales de innovación en América Latina. Washington DC: Banco Interamericano de Desarrollo.

Marquina, M. y Chiroleu, A. (2015). ¿Hacia un nuevo mapa universitario? La ampliación de la oferta y la inclusión como temas de agenda de gobierno en Argentina. Propuesta Educativa, 43, pp. 7-16.

MINCyT (2011). Hacia una Argentina Innovadora: Plan Nacional de Ciencia, Tecnología e Innovación. Lineamientos 2012-2015. Buenos Aires: Ministerio de Ciencia, Tecnología e Innovación Productiva (MINСуT).

MINCyT (2013). Argentina Innovadora 2020: Plan Nacional de Ciencia, Tecnología e Innovación. Lineamientos Estratégicos 2012-2015. Buenos Aires: Ministerio de Ciencia, Tecnología e Innovación Productiva (MINCyT). Disponible en: https://www.argentina. gob.ar/sites/default/files/pai2020.pdf.

Moldovan, P., Gordon, A. y Di Marzo, E. (2011). Estructura científica y perfil tecnoproductivo de la Argentina. En F. Porta y G. Lugones (Eds.), Investigación científica e innovación tecnológica en la Argentina: Impacto de los fondos de la Agencia Nacional de Promoción Científica y Tecnológica. Bernal: Editorial UNQ.

Niembro, A. (2019). Problemas y necesidades de los sistemas regionales de innovación en Argentina: Hacia un enfoque territorial de las políticas de CTI.
REDES - Revista de Estudios Sociales de la Ciencia y la Tecnología, 25(48), próximo a publicarse en versión electrónica.

Niembro, A. (2020). ¿Federalización de la ciencia y tecnología en Argentina? La carrera del investigador de CONICET (2010-2019). Ciencia, Docencia y Tecnología, 31(60), pp. 1-33..

Niosi, J. (2013). La construcción de sistemas nacionales para la innovación: Un análisis comparativo entre Argentina y Canadá. En G. Dutrénit y J. Sutz (Eds.), Sistemas de innovación para un desarrollo inclusivo: La experiencia latinoamericana. México DF: LALICS.

ONP (2016). Instituto Nacional de Tecnología Industrial. Política presupuestaria de la entidad. Oficina Nacional de Presupuesto (ONP), Ministerio de Economía de la Nación. Disponible en: https://www. minhacienda.gob.ar/onp/documentos/presutexto/ proy2016/jurent/docs/P16E608.rtf.

Peirano, F. (2011). El FONTAR y la promoción de la innovación en empresas entre 2006 y 2010. En F. Porta y G. Lugones (Eds.), Investigación científica e innovación tecnológica en la Argentina: impacto de los fondos de la Agencia Nacional de Promoción Científica y Tecnológica. Bernal: Editorial UNQ.

Rivas, G., Rovira, S. y Scotto, S. (2014). Reformas a la institucionalidad de apoyo a la innovación en América Latina: Antecedentes y lecciones de estudios de caso. En G. Rivas y S. Rovira (Eds.), Nuevas instituciones para la innovación: Prácticas y experiencias en América Latina. Santiago de Chile: CEPAL, Naciones Unidas.

Rodríguez Reyes, C. y Meza Medina, G. (noviembre, 2011). Federalización educativa en México... ¿y la educación? Trabajo presentado en XI Congreso Nacional de Investigación Educativa. Consejo Mexicano de Investigación Educativa (COMIE), México DF. 
Disponible en: http://www.comie.org.mx/congreso/ memoriaelectronica/v11/docs/area_13/1785.pdf

Ruarte Bazán, R. (septiembre, 2009). Hacia una interpretación del federalismo argentino actual. Trabajo presentado en 42 Jornadas Internacionales de Finanzas Públicas. Universidad Nacional de Córdoba, Córdoba. Disponible en: https://rdu.unc.edu.ar/ handle/11086/6025

Sarthou, N. (2019). Tendencias en la evaluación de la ciencia en Argentina: Género, federalización y temas estratégicos. Ciencia, Docencia y Tecnología, 30(59), pp. 37-73.

SECYT (2005). Bases para un Plan Estratégico de Mediano Plazo en Ciencia, Tecnología e Innovación. Buenos Aires: Secretaría de Ciencia, Tecnología e Innovación Productiva (SECyT).

SECyT (2019). Estrategia Nacional de CTI Argentina Innovadora 2030. Buenos Aires: Secretaría de Gobierno de Ciencia, Tecnología e Innovación Productiva (SECYT). Mimeo.

Solanas, C. (mayo, 2009). El nuevo INTI y el territorio. Trabajo presentado en $\mathrm{V}$ Congreso Argentino de Administración Pública. Asociación Argentina de Estudios de Administración Pública, San Juan. Presentación disponible en: http://www.asociacionag.org. ar/pdfcap/5/SOLANAS,\%20CRISTINA\%20-\%20 LA\%2OFEDERALIZACION\%2ODEL\%2OINTI.ppt.

Suárez, D. y Fiorentin, F. (2018). Federalización y efecto Mateo en la política científica: El caso del PICT en la Argentina (2012-2015). Documento de Trabajo No. 12. Buenos Aires: Centro Interdisciplinario de Estudios en Ciencia, Tecnología e Innovación (CIECTI).

Szpeiner, A. y Jeppesen, C. (septiembre, 2013). Políticas de federalización en ciencia y tecnología: Avances en el CONICET. Trabajo presentado en VII Congreso Argentino de Administración Pública. Asociación Argentina de Estudios de Administración Pública, Mendoza. Disponible en: https://aaeap.org.
ar/wp-content/uploads/2018/11/Szpeiner_Alfonsina_ Jeppesen_Cynthia_Politicas_de_Federalizacion_en_ Ciencia_y_Tecnologia_Panel_066.pdf

Yoguel, G., Borello, J. y Erbes, A. (2005). Sistemas locales de innovación: Los casos de Córdoba, Rafaela, Rosario y Tucumán, Salta y Jujuy. Documento de Proyecto. Buenos Aires: Observatorio Nacional de Ciencia, Tecnología e Innovación Productiva.

Zurbriggen, C. y González Lago, M. (2010). Políticas de Ciencia, Tecnología e Innovación en los países del MERCOSUR. Montevideo: Centro de Formación para la Integración Regional. 